\title{
SAND2000-0733J
}

\section{Liquid/Vapor Surface Tension of Metals: Embedded Atom Method with Charge Gradient Corrections}

\author{
Edmund B. Webb III and Gary S. Grest \\ Sandia National Laboratory, Albuquerque, New Mexico 87185-1411
}

(March 14, 2000) \begin{abstract}
Molecular dynamics (MD) simulations for three separately parameterized embedded atom method
(EAM) function sets are used to determine the liquid/vapor surface tension $\gamma$ for $\mathrm{Al}, \mathrm{Ni}, \mathrm{Cu}, \mathrm{Ag}$, and $\mathrm{Au}$. The three EAM models differ in both the functional forms employed and the fitting $\boldsymbol{C}$ procedure used. All the EAM potentials underestimate $\gamma$ but one of the models performs consistently better than the others. We show that including a correction to the local charge density associated with gradients in the density together with exploiting the invariance of the EAM bulk potential to appropriate transformations in the charge density can lead to improved values-for $\gamma$, as well as for solid free surface energies, within existing EAM function sets.
\end{abstract}

Using atomistic simulations to study physical processes on surfaces calls for length and time scales which are large relative to the complexity of the corresponding calculations. Thin film growth, liquid alloy wetting and spreading, and corrosion are phenomena related to many technologies which stand to benefit from the knowledge gained via large scale atomistic surface simulations. Computational resources are increasing and such calculations are now feasible so greater scrutiny is being applied to the performance of existing interatomic potentials at predicting surface energetics of materials. In many instances, the scale of simulation necessary precludes the use of first principles calculations so there is a need for accurate empirical interatomic potential functions which can be applied via classical Monte Carlo (MC) or molecular dynamics (MD) simulations.

A popular choice of interatomic potential for simulating metals is the embedded atom method (EAM) which employs a multibody contribution to the binding energy of an atom that has been demonstrated to overcome deficiencies in pair potentials used for simulating metals [1]. The EAM has physical origins in density functional theory but is an empirical potential which is only slightly more difficult to implement than a pair potential. A large body of prior work exists applying the EAM to properties of metals including bulk, surface, point defect, and alloy behavior [2]. This model has also been used to study properties of liquid metals [3-5] as well as dynamic processes such as deposition [6] and indentation [7]. One shortcoming of many parameterizations for metals within the EAM is the underprediction of surface energetics in both the solid [2] and liquid [3] states. Some part of this deficiency, however, is directly related to the fitting procedure used when creating an EAM function set which accounts for some models performing better than others.

The energy of a system of $N$ atoms in the EAM is

$$
E_{s}=\sum_{i=1}^{N}\left[F_{i}\left(\rho_{i}\right)+\frac{1}{2} \sum_{j \neq i} \phi_{i j}(R)\right] .
$$

Here $\rho_{i}$ is the local charge density at atom $i$,

$$
\rho_{i}=\sum_{j \neq i} \rho_{j}{ }^{a}(R)
$$

where $\rho_{j}{ }^{a}(R)$ is the spherically symmetric electron density contributed by atom $j$ at a distance $R$ from atom $i$. The embedding term $F_{i}\left(\rho_{i}\right)$ is the energy associated with embedding atom $i$ into an electron density $\rho_{i}$ and $\phi_{i j}(R)$ is a pair potential between atoms $i$ and $j$ separated by distance $R$. An EAM function set for a single metal is composed of $\rho_{j}{ }^{a}(R), \phi_{i j}(R)$, and $F_{i}\left(\rho_{i}\right)$. There is no single way to determine these functions but a typical approach is to assume some physically appropriate mathematical forms and fit the resulting parameters to experimentally measured material properties. The cutoff for $\rho_{j}{ }^{a}(R)$ and $\phi_{i j}(R)$ is also a fitting parameter, whose value is typically from one to five neighbor shells in the fcc crystal. Many function sets have been developed employing only bulk data. If surface data was included, it was not highly weighted in the fitting. This is one reason why most of these parameterizations do not perform well when used to quantitatively predict surface properties where the charge densities are significantly different from those in the bulk crystal.

One way of improving EAM surface energetic predictions is to fit new potential functions to available surface experimental data relevant to the intended application of the model. This, however, runs the risk of achieving less success in bulk thermodynamic predictions and entails the obvious labor associated with creating potentials. With the plethora of EAM functions in the literature, it is useful to investigate ways of reconciling their surface prediction accuracy. One method that has been proposed to achieve this [8] and demonstrated to work for the (110) surface reconstruction of $\mathrm{Au}[9]$ involves adding corrections to the argument of the embedding function related to the local gradient, or nonuniformity, in the charge density:

$$
F_{i}\left(\rho_{i}+\alpha \nabla^{2} \rho_{i}+\beta\left|\nabla \rho_{i}\right|^{2}\right) .
$$


Density functional theory demonstrates that these are the lowest order terms related to charge nonuniformity upon which the embedding energy depends [8]. Because such corrections are zero in the equilibrium crystal, they do not alter bulk calculations and the parameters $\alpha$ and $\beta$ can be fit to relevant experimental surface data. For $\mathrm{Au}$, Roelofs et al. [9] found that they were able to correct inaccurate predictions of the relative stability of (110) surface reconstructions using this approach. They assumed $\alpha=0$ since gradients near surfaces are expected to be fairly significant. Additionally, the EAM cohesive energy is invariant under the transformations:

$$
\begin{aligned}
F_{i, c}\left(\rho_{i}\right) & =F_{i}\left(\rho_{i}\right)+c \rho_{i} \\
\phi_{i j, c}(R) & =\phi_{i j}(R)-c \rho_{i}{ }^{a}(R)-c \rho_{j}{ }^{a}(R),
\end{aligned}
$$

where $c$ is an arbitrary constant. Roelofs et al. point out that exponential functions were used for $\rho_{i}{ }^{a}(R)$ in their work and, as such, the Laplacians are proportional to the charge densities and would therefore represent such a transformation. They also point out that these transformed functions respond differently to the correction term $\beta\left|\nabla \rho_{i}\right|^{2}$ and suggest this as an additional route to improve the treatment of surface properties [9].

Herein we test twelve existing EAM function sets for their ability to predict liquid/vapor surface tension $\gamma$. In all cases, we find results that are less than experiment. We investigate correcting this deficiency by applying Eq. [3] and demonstrate that the degree of success obtained depends on the shape of $F_{i}\left(\rho_{i}\right)$. We then apply Eq. [4] to alter the shape of the embedding functions in an attempt to further improve surface predictions through Eq. [3]. Solid free surface energies from the models are demonstrated to benefit from this same approach.

The EAM function sets used are due to Foiles et al. [10,11], Voter and Chen [12], and Mishin et al. [13]; we refer to them as FBD, VC and MFMP, respectively [14]. For FBD and VC, there are function sets for $\mathrm{Al}, \mathrm{Ni}, \mathrm{Cu}$, $\mathrm{Ag}$, and $\mathrm{Au}$; for MFMP there are sets for $\mathrm{Al}$ and $\mathrm{Ni}$. The FBD functions were fit to experimental data associated with charge densities near bulk equilibrium and $\phi_{i j}(R)$ is purely repulsive. The VC functions were fit similarly but with the inclusion of the diatomic molecule strength. $\phi_{i j}(R)$ in the VC model is a Morse function with an attractive regime. The MFMP functions were formed from arbitrary cubic splines and the fitting employed a testing phase that involved $a b$ initio calculated energetics of structures with charge densities far from bulk equilibrium. The methodology used by the three groups to arrive at their function sets was sufficiently different to suspect that differences in $\gamma$ prediction could be related to aspects of the potential creation.

Semi-periodic liquid slabs of 3200 atoms were maintained at constant $T$ with a Berendsen thermostat [15] and a time step of $0.5 \mathrm{fs}$. Starting from an fcc crystal, the slabs were melted and equilibrated for at least $1 \mathrm{~ns}$.
Other temperatures were studied by reequilibrating the system for $250 \mathrm{ps}$. Data runs $1 \mathrm{~ns}$ in duration were then carried out for each $T$ of interest. The mechanical definition was used to calculate $\gamma$ and the simulation durations were long enough that statistical error was below $5 \%$ for all $\gamma$. Solid free surface energies were calculated by allowing the relevant surface to relax at $T=5 \mathrm{~K}$ for $50 \mathrm{ps}$ and then gathering statistics over another $50 \mathrm{ps}$. Comparisons between some of our results and prior solid surface calculations $[10,16]$ indicate this method is sufficient for our purposes. By using a time average of the local charge gradient that was updated every 2000 steps, the gradient in the bulk region was negligible compared to the local charge density.

Figure 1 is a plot of $\gamma$ for $\mathrm{Al}, \mathrm{Ni}, \mathrm{Cu}, \mathrm{Ag}$, and $\mathrm{Au}$; results from the simulations as well as experiment [17] are shown. Only for Al in the VC model is there good agreement with experiment. In almost all the cases studied, the experimental surface tension is underestimated by $20 \%$ to $60 \%$. While ambiguity exists in the experimental literature for $\gamma$, the magnitude of this discrepancy is, in most cases, too large to attribute to anything but deficiencies in the models. However, qualitative features of the predictions agree well with experiment: the ordering of metals by $\gamma$ is correct in all models and there is little dependence of $\gamma$ on $T$. In all cases, the VC model performs best, followed by the MFMP model, and then the FBD model. Considering the narrow range of charge density probed by the function fitting for FBD, it is not surprising that the energy of a structure far from the bulk fcc crystal, such as a liquid surface, would not be described well by this model. Both the VC and the MFMP models employ a wider range of charge density states in their function fitting which at least partly explains their better performance.

To examine the effect of adding charge density gradient contributions to the argument of the embedding function, $\mathrm{Ni}$ and $\mathrm{Au}$ in both the FBD and VC models were studied. As in prior work [9], we set $\alpha=0$ and study the effect of varying $\beta$. Observation of Eq. [3] shows that the argument to the embedding function is increased for positive charge gradient corrections $(\beta>0)$. For $\mathrm{Au}$ in the VC model, Roelofs et al. identified a range of $\beta$ $(0.6 \lesssim \beta \lesssim 1.2)$ which was physically reasonable based on the relative stability of the $(1 \times 1),(1 \times 2)$, and (1 $x 3)$ reconstructions of the (110) surface. To more thoroughly examine the effect of this correction, we present in Fig. 2 results for $\gamma$ versus $\beta$ for $\beta=-2.0$ to 2.0. Data for both $\mathrm{Ni}$ and $\mathrm{Au}$ show that positive charge gradient corrections improve the prediction of $\gamma$ for the $\mathrm{VC}$ model. For VC Au within the physically reasonable range of $\beta$ the model underpredicts experiment by $25 \%$. Extending $\beta$ to 2.0 reduces the deviation between experiment and model but not very much. For Ni in the VC model, however, $\gamma$ increases much more rapidly with $\beta$ so that for $\beta=0.5$ the deviation between model and experiment is 


\section{DISCLAIMER}

This report was prepared as an account of work sponsored by an agency of the United States Government. Neither the United States Government nor any agency thereof, nor any of their employees, make any warranty, express or implied, or assumes any legal liability or responsibility for the accuracy, completeness, or usefulness of any information, apparatus, product, or process disclosed, or represents that its use would not infringe privately owned rights. Reference herein to any specific commercial product, process, or service by trade name, trademark, manufacturer, or otherwise does not necessarily constitute or imply its endorsement, recommendation, or favoring by the United States Government or any agency thereof. The views and opinions of authors expressed herein do not necessarily state or reflect those of the United States Government or any agency thereof. 


\section{DISCLAIMER}

Portions of this document may be illegible in electronic image products. Images are produced from the best available original document. 
less than $10 \%$. Considering the variation in experimental data, this is acceptable. Increasing $\beta$ for the FBD model is seen to steadily decrease $\gamma$. Negative charge gradient corrections improve $\gamma$ predictions for both $\mathrm{Au}$ and $\mathrm{Ni}$ in the FBD model. At $\beta=-2.0$ for $\mathrm{Ni}$ and -1.5 for $\mathrm{Au}$, the deviation between model and experiment has been reduced but is still $40 \%$ (the Au system was unstable for $\beta \lesssim-1.5$ ). While data for one temperature is shown, similar results for both models were obtained upon changing $T$.

The intent of the charge density correction term is to place a penalty on the presence of charge gradients so that defect structures, like surfaces, would have a higher energy. With the arbitrary nature of EAM functions, however, it is not implicit which direction one should shift along $F_{i}\left(\rho_{i}\right)$ to cause an energetic penalty. Observation of the embedding functions and identification of the approximate value of charge density for atoms in the bulk and surface clarifies this. While it is true that the charge goes from a bulk liquid value to zero through the surface region, atoms in the surface experience, on average, a charge density no less than about $40 \%$ of the bulk value. As such, identifying a relevant range in $\rho_{i}$ on $F_{i}\left(\rho_{i}\right)$ is straightforward. At the very least, the upper limit of this range can be taken fairly literally. Figure 3 shows $F_{i}\left(\rho_{i}\right)$ for $\mathrm{Ni}$ and $\mathrm{Au}$ for both the FBD and VC models. For Ni in the FBD model and Au in both models, there are also transformed versions of each function $F_{i, c}\left(\rho_{i}\right)$ which will be discussed below; the untransformed functions are shown with solid curves. The relevant range of $\rho_{i}$ in a liquid surface simulation for each model is indicated with bars at the top of the figures. The difference in charge density range for the $\mathrm{FBD}$ and $\mathrm{VC}$ models is apparent. For both metals in the VC model, the relevant range of charge density is a region of positive slope in $F_{i}\left(\rho_{i}\right)$. The opposite is true for both metals in the FBD model. Therefore, for the VC model, an increase in energy is achieved by increasing the argument to the embedding function via positive values of $\beta$. For the FBD model, an energetic penalty is achieved by shifting in the opposite direction, that is, for $\beta<0$.

The change in surface tension with $\beta$ is dependent upon both the magnitude of gradients in the surface and the local slope of $F_{i}\left(\rho_{i}\right)$. Eq. [4] allows one to alter the slope of $F_{i}\left(\rho_{i}\right)$ giving $F_{i, c}\left(\rho_{i}\right)$. As discussed above, the sign of $\beta$ which achieves an increase in $\gamma$ is dependent upon the sign of the slope of $F_{i}\left(\rho_{i}\right)$. As such, a greater increase may be realized if the steepness of $F_{i}\left(\rho_{i}\right)$ is greater, that is, if the magnitude of the slope is increased. For $\mathrm{VC}$ functions with positive slope, this means using $c>0$ while the opposite is true for FBD. This is further emphasized in Fig. 3 where we present transformed embedding functions $F_{i, c}\left(\rho_{i}\right)$ for $\mathrm{Ni}$ in the FBD model and $\mathrm{Au}$ in both the FBD and VC models. The short and long dashed curves are for $c=-100$ and $c=25$, respectively (only $F_{i, c}\left(\rho_{i}\right)$ for $c=25$ is shown for $\mathrm{VC} \mathrm{Au}$ ). To demonstrate the effect of combining Eq. [3] with Eq. [4], in Fig. 2 we present $\gamma$ versus $\beta$ obtained using transformed function sets (solid symbols). For $\mathrm{Au}$ and $\mathrm{Ni}$ in the FBD model we use $c=-100$ and for $\mathrm{Au}$ in the $\mathrm{VC}$ model we use $c=25$ (since $\gamma$ for $\mathrm{Ni}$ in the VC model responded so strongly to $\beta$ for $c=0$, we $\operatorname{did}$ not experiment with transforming that function set). Results for $\gamma$ from transformed functions are identical to those from untransformed functions for $\beta=0$. For $\mathrm{Au}$ in the VC model, $F_{i, c}\left(\rho_{i}\right)$ results in a stronger response of $\gamma$ to $\beta$ such that, for $\beta=2.0$, the model predicts $\gamma$ within $5 \%$ of experiment. For both metals in the FBD model, the success is much less. In fact, $A u$ in the transformed FBD model has a very limited range of $\beta$ for which the system is stable and, within that range, there is no improvement of $\gamma . F_{i, c}\left(\rho_{i}\right)$ for FBD Ni does not suffer from the same instability problems as $A u$ in the range of $\beta$ studied but for $\beta=-2.0$, the model still underpredicts experiment by $30 \%$. The FBD model employs a purely repulsive pair term so that all attraction is provided by the embedding term. Furthermore, the range of charge density accessed by the model is narrow and close to 0 . These two attributes combine so that, when using negative charge gradient contributions, the embedding term will eventually go to zero resulting in system instability. This also places a very obvious upper limit on the increase in $\gamma$ that can be achieved with this method. The VC model is both more robust and responsive to the combination of transforming the functions and adding charge gradient contributions. Fig. 3 demonstrates that the embedding function for $\mathrm{VC} \mathrm{Au}$ is more analogous after transformation to $F_{i}\left(\rho_{i}\right)$ for $\mathrm{VC} \mathrm{Ni}$. Two observations can be made: 1) EAM models which employ a wider range of charge density respond better to the inclusion of charge gradient contributions and 2) the response of surface energetics to these corrections is most desirable when the model is operating in a range of $\rho_{i}$ where $F_{i}\left(\rho_{i}\right)$ has positive and appreciable slope. While a large value of $c$ could be used to create transformed FBD embedding functions with such a slope, the narrow range of $\rho_{i}$ accessed might still limit the success of this method. While detailed discussion must be reserved for a forthcoming publication, results obtained from FBD and VC models for other metals as well as from the MFMP model further bear out these observations.

Ref. [9] demonstrates that low temperature surface energies for reconstructions of $\mathrm{Au}$ (110) in the $\mathrm{VC}$ model order properly only within a narrow region of $\beta$ at $c=0$. It is therefore important to calculate solid free surface energies using charge gradient corrections and, where applicable, transformed function sets. A requirement for all models is that the appropriate stability of the $(1 \times 1)$ and ( $1 \times 2)$ reconstructions of the (110) surface is predicted. Additionally, it is required that proper energetic ordering of the (100), (110), and (111) surfaces is predicted. Thorough discussion of solid surface data must also be 
reserved but the only limitation these requirements place on the current results is for VC Au. Similar to Roelofs et al. for $c=0$, we find (for $c=25)$ that the $(1 \times 1)$ reconstruction of the $\mathrm{Au}$ (110) surface becomes stable relative to $(1 \times 2)$ between $\beta=1.0$ and 1.5. However, this still corresponds with significant improvement over the untransformed function set. Furthermore, we have not performed a search for optimized combinations of $c$ and $\beta$. A final point about solid surface data is that similar improvement in the magnitude of solid free surface energies was found as for liquid/vapor surface tension. Clearly, it is desirable to use the same value of $c$ and $\beta$ across temperature and phase space and results obtained demonstrate this is possible.

The use of charge gradient corrections in conjunction with appropriate transformations of EAM function sets can provide surface energy predictions in excellent agreement with experiment for solid and liquid phase metals. The degree of success obtained depends on the shape of embedding functions within the model as well as the range of charge density over which the model operates. This extension of the EAM is only marginally more difficult to implement than a standard EAM and can produce empirical interatomic functions with excellent quantitative surface predictions.

We acknowledge Mike Baskes and Stephen Foiles for many helpful conversations and code assistance. Sandia is a multiprogram laboratory operated by Sandia Corporation, a Lockheed Martin Company, for the United States Department of Energy under Contract DE-AC0494AL85000.

[1] M. S. Daw and M. I. Baskes, Phys. Rev. B 29, 6443 (1984).

[2] M. S. Daw, S. M. Foiles, and M. I. Baskes, Mater. Sci. Rep. 9, 251 (1993).

[3] L. M. Holzman, J. B. Adams, S. M. Foiles, and W. N. G. Hitchon, J. Mater. Res. 6, 298 (1991).

[4] B. Sadigh and G. Grimvall, Phys. Rev. B 54, 15742 (1996).

[5] M. Asta et al., Phys. Rev. B 59, 14271 (1999).

[6] E. B. W. III and S. H. Garofalini, Surf. Sci. 319, 381 (1994).

[7] C. L. Kelchner, S. J. Plimpton, and J. C. Hamilton, Phys. Rev. B 58, 11085 (1998).

[8] M. S. Daw, Phys. Rev. B 39, 7441 (1989).

[9] L. D. Roelofs, S. M. Foiles, M. S. Daw, and M. I. Baskes, Surf. Sci. 234, 63 (1990).

[10] S. M. Foiles, M. I. Baskes, and M. S. Daw, Phys. Rev. B 33, 7983 (1986).

[11] S. M. Foiles and M. S. Daw, J. Mater. Res. 2, 5 (1987).

[12] A. F. Voter, Los Alamos Unclassified Technical Report LA-UR 93-3901 (1993); A. F. Voter and S. P. Chen,
Mater. Res. Soc. Proc. 82, 175 (1987).

[13] Y. Mishin, D. Farkas, M. J. Mehl, and D. A. Papaconstantopoulos, Phys. Rev. B 59, 3393 (1999).

[14] The potential for $\mathrm{Al}$ that we refer to as FBD was due to Foiles and Daw [11] and was not parameterized for alloy calculations like the potentials in [10]. However, the methodology for creating the $\mathrm{Al}$ potential was nearly the same so, for convenience, the label FBD was used.

[15] M. P. Allen and D. J. Tildesley, Computer Simulation of Liquids (Clarendon, Oxford, 1987).

[16] S. P. Chen and A. F. Voter, Surf. Sci. Lett. 244, L107 (1991).

[17] R. C. Weast, Handbook of Chemistry and Physics, 61st Edition (CRC Press, Boca Raton, FL, 1980). 


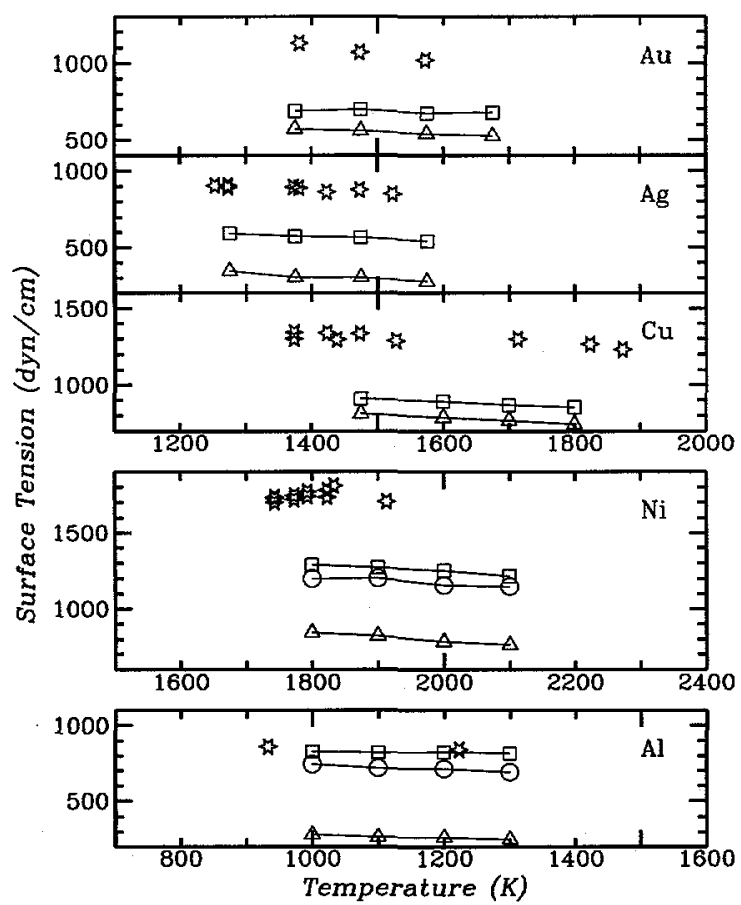

FIG. 1. Surface tension versus $T$ for $\mathrm{Al}, \mathrm{Ni}, \mathrm{Cu}, \mathrm{Ag}$, and Au. Data are shown for FBD (triangles), MFMP (circles), and VC (squares) models as well as experiment [17] (stars).

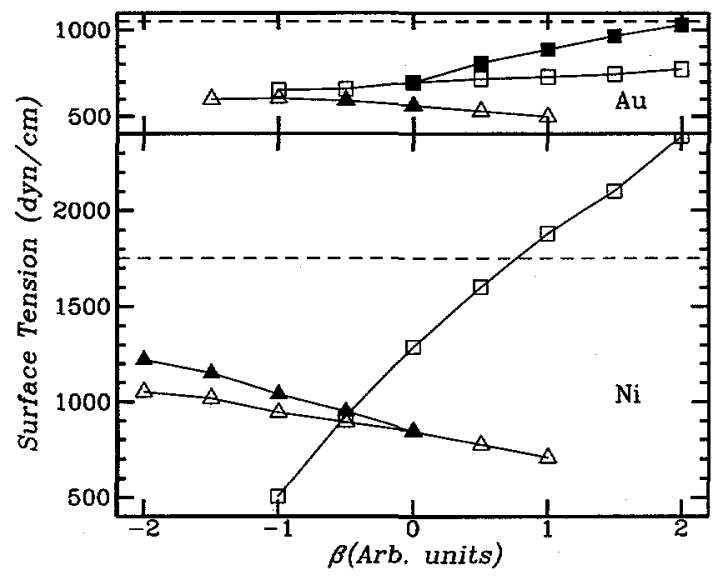

FIG. 2. Surface tension versus $\beta$ for $\mathrm{Ni}(T=1800 K)$ and $\mathrm{Au}(T=1475 K)$ for the FBD (triangles) and VC (squares) models. Open symbols show data for $c=0$; closed symbols show data for transformed functions for $\mathrm{Au}$ in both FBD $(c=-100)$ and $\mathrm{VC}(c=25)$ models and for $\mathrm{Ni}$ in FBD $(c=-100)$. The horizontal dashed lines on each plot indicate the experimental value of $\gamma$.
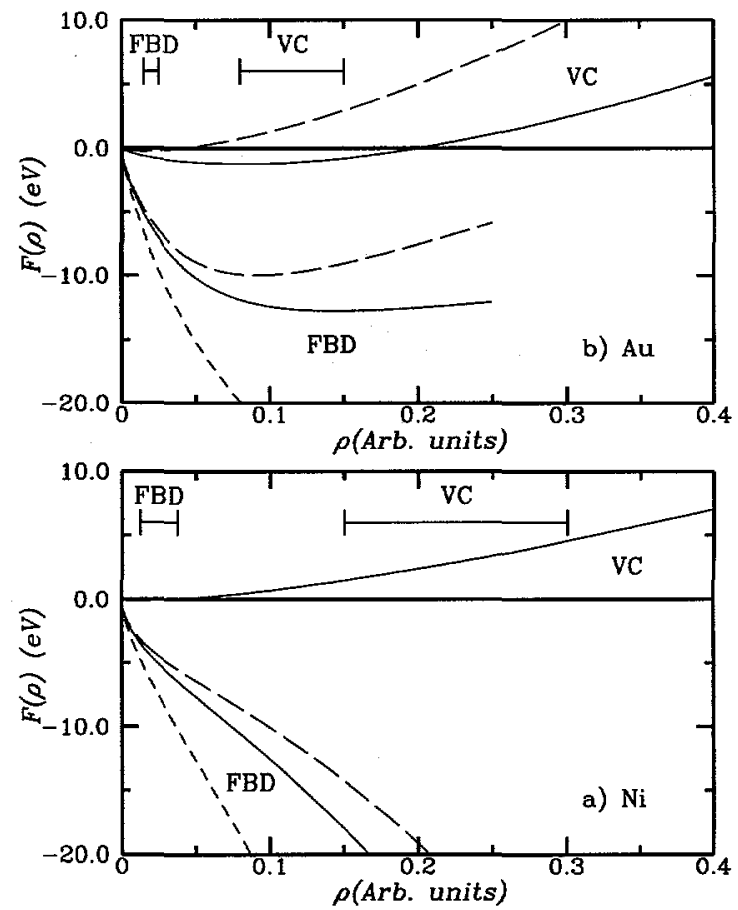

FIG. 3. The embedding functions for $c=0$ (solid), -100 (short dash), and 25 (long dash) for a) $\mathrm{Ni}$ and b) Au. Data from the FBD model are shown in the lower curves and VC in the upper curves. Horizontal hash marks at the top of each figure show the relevant range of $\rho_{i}$ in the liquid phase for each model. 